\title{
Social Selling - Buzzword oder innovativer Vertriebsansatz?
}

„Social Selling wird als Allheilmittel für schnelle Umsatzsteigerungen propagiert.“ (Schmäh et al. 2016, S. 15).

„Social Selling auf LinkedIn ist ein Marathon und kein Sprint!“ (Behrens 2020)

„Der Vertrieb wird regelmäßig von Methoden-Hypes heimgesucht. [...] So entstehen immer neue Vertriebsmethoden, mit denen große Versprechen verbunden werden. Der aktuelle Schlüssel zum Erfolg heißt Social Selling.“ (Kords 2020)

Die Überschrift des einleitenden Kapitels und die anfänglichen Zitate sollen die kritisch-neutrale Perspektive dieses Werkes verdeutlichen. Unbestritten ist sicherlich, dass sich das Mediennutzungsverhalten der Menschen kontinuierlich verändert und dass der Nutzung sozialer Medien in all deren unterschiedlichen Formen insgesamt ein wachsender Stellenwert attestiert werden kann. Konsumenten informieren sich schon lange und in weiter zunehmendem Maße in sozialen Medien über Produkte und Unternehmen. Bereits im Jahr 2009 überstieg der durch die Nutzung von sozialen Netzwerken entstandene Traffic den durch EMails verursachten Datenverkehr mit weiterhin zunehmender Tendenz (Ahearne und Rapp 2010, S. 112; Moore et al. 2015, S. 1). Im Jahr 2020 nutzten ca. 4,0 Mrd. Menschen weltweit, sprich etwa die Hälfte der Weltbevölkerung, soziale Medien aktiv. Die Nutzerzahl erhöht sich ständig: Im letzten Jahr kamen 376 Mio. neue Social-Media-Nutzer (+11\%) dazu (Kemp 2020, S. 7 ff.).

Die Vielfalt der sozialen Medien zeigt das Social Media Prisma (Franke und ethority 2021) in Abb. 1.1. Insbesondere die „Professional Networks“ sind für Social Selling im B2B-Kontext interessant. Auch Plattformen, die dazu dienen, 


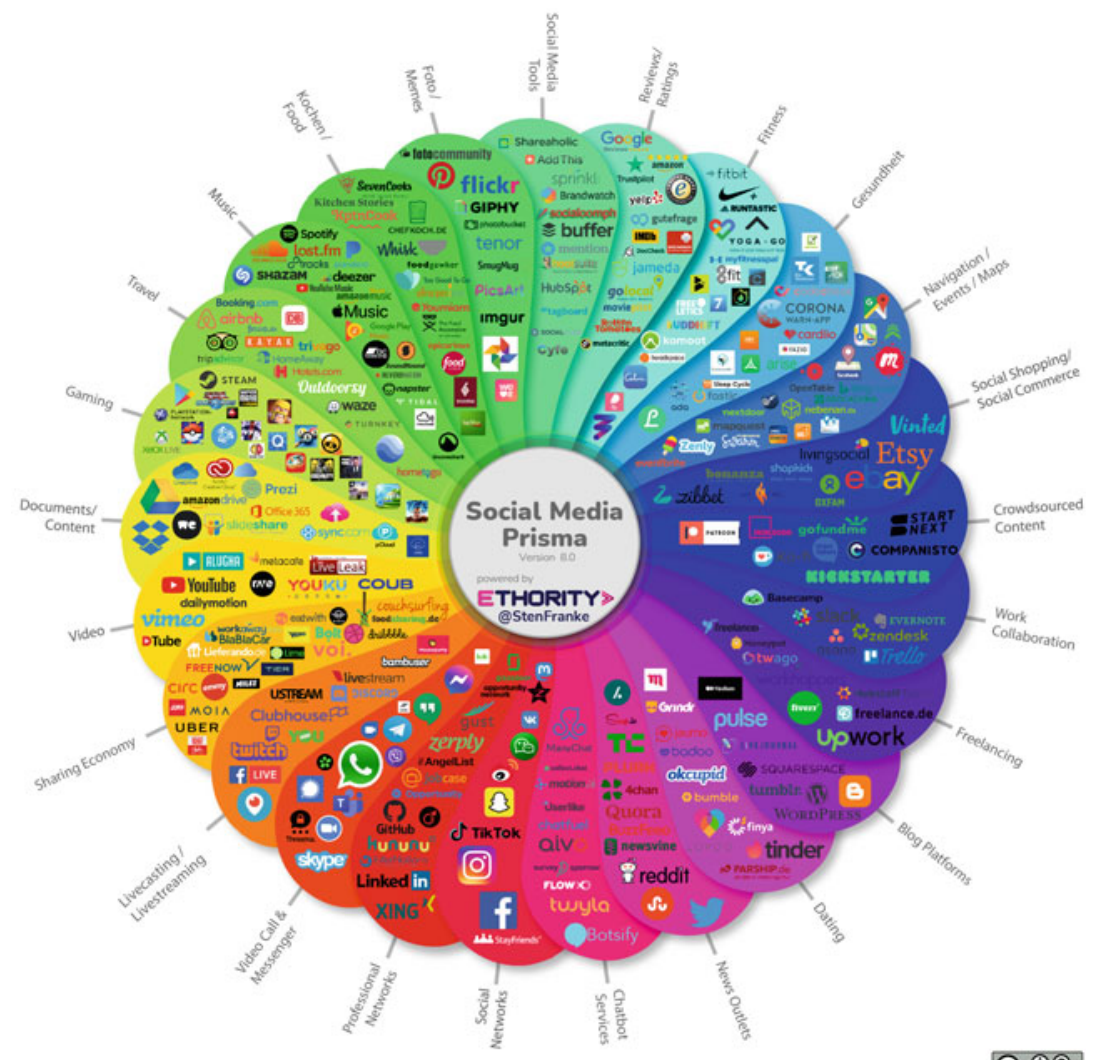

Abb. 1.1 Social Media Prisma (Franke und ethority 2021) 
sich als Experte mit Hilfe von Inhalten zu positionieren, können für SocialSelling-Zwecke genutzt werden (z. B. Twitter, Instagram, diverse Blogging-, Video-, Bilder- oder Content-Plattformen).

Aktuell wird Social Selling (besonders im B2B-Sales-Kontext) als besonders heißes Thema in der Praxis diskutiert, wie es mit "neuen“ Trends und Buzzwords häufig im Marketing der Fall ist. In der Wissenschaft wurde schon vor einigen Jahren verkündet, dass Social Selling eine „,neue Ära“ einläutet, in der das professionelle Verkaufen auf der Stärke von Social Media gründet (Agnihotri et al. 2012, S. 341). Allerdings erinnerten die Berater von McKinsey \& Company bereits im Jahr 2013 daran, dass es beim Verkauf um mehr gehen sollte als nur um den Verkauf von Dingen. Beim Verkauf ginge es darum, Kunden zu verstehen, mit ihnen zu interagieren und ihnen zu helfen. Soziale Netzwerke sind hierbei wichtige Instrumente, um diese Dinge zu tun und im Verkaufszyklus zu helfen (McKinsey \& Company 2013). Genau deshalb können Social-Selling-Ansätze einen großen Mehrwert für den Vertrieb schaffen.

Auf Basis der aktuellen Relevanz des Themas „Social Selling“ in Praxis und Wissenschaft soll im weiteren Verlauf geklärt werden, ob es sich nun um ein Buzzword, respektive eine Modeerscheinung handelt oder einen wirklich radikal innovativen Vertriebsansatz. Dazu wird zunächst der aktuelle State of the Art (Begriff, Einordnung ins Vertriebsmanagement, Stand der wissenschaftlichen Forschung sowie der Nutzen- und Wertbeitrag) analysiert. Anschließend werden spezifische Aspekte, die im Kontext des Social Sellings relevant sind, vorgestellt (Content Marketing, Personal Branding, Employee Advocacy, Influencer Marketing im B2B-Kontext, Social Listening). Weiterhin werden Praxisherausforderungen, typische Plattformen und Tools sowie Risiken des Social Sellings diskutiert. Schließlich wird versucht, die Frage aus der Überschrift dieses Kapitels zu beantworten und einen Ausblick zu wagen. 
Open Access Dieses Kapitel wird unter der Creative Commons Namensnennung 4.0 International Lizenz (http://creativecommons.org/licenses/by/4.0/deed.de) veröffentlicht, welche die Nutzung, Vervielfältigung, Bearbeitung, Verbreitung und Wiedergabe in jeglichem Medium und Format erlaubt, sofern Sie den/die ursprünglichen Autor(en) und die Quelle ordnungsgemäß nennen, einen Link zur Creative Commons Lizenz beifügen und angeben, ob Änderungen vorgenommen wurden.

Die in diesem Kapitel enthaltenen Bilder und sonstiges Drittmaterial unterliegen ebenfalls der genannten Creative Commons Lizenz, sofern sich aus der Abbildungslegende nichts anderes ergibt. Sofern das betreffende Material nicht unter der genannten Creative Commons Lizenz steht und die betreffende Handlung nicht nach gesetzlichen Vorschriften erlaubt ist, ist für die oben aufgeführten Weiterverwendungen des Materials die Einwilligung des jeweiligen Rechteinhabers einzuholen. 